\title{
LIMB SALVAGE USING DISTRACTION OSTEOGENESIS
}

\author{
A CLASSIFICATION OF THE TECHNIQUE
}

HIROYUKI TSUCHIYA, KATSURO TOMITA, KOJI MINEMATSU, YOSHIHIKO MORI, NAOHIRO ASADA, SHINJI KITANO

From Kanazawa University, Japan

We report the results of distraction osteogenesis (callotasis) for the reconstruction of extensive defects after the excision of skeletal tumours in the limbs. Bone transport was performed in ten patients (five osteosarcomas and five giant-cell tumours), shorteningdistraction in three (two osteosarcomas and one Ewing's sarcoma), and distraction osteogenesis combined with an intramedullary nail to reduce the time of external fixation in six (three osteosarcomas, two chondrosarcomas, and one malignant fibrous histiocytoma).

The mean length of the defects after excision of the lesion was $8.4 \mathrm{~cm}$. The mean external fixation index was $39.5 \mathrm{days} / \mathrm{cm}$ for the group treated by bone transport, $34.1 \mathrm{days} / \mathrm{cm}$ for the shortening-distraction group, and $24.0 \mathrm{days} / \mathrm{cm}$ for the group treated by distraction and an intramedullary nail. Functional evaluation gave excellent results in 12 patients, good in five and fair in two. There were ten complications in 19 patients, all of which were successfully treated.

We also classified reconstruction using distraction osteogenesis into five types based on the location of the defects after resection of the tumour: type 1, diaphyseal; type 2, metaphyseal; type 3, epiphyseal; type 4, subarticular reconstruction; and type 5, arthrodesis.

Our results suggest that reconstruction using distraction osteogenesis provides bone which will develop sufficient biomechanical strength and durability. It is beneficial in patients with an expectation of long-term survival and in growing children.

J Bone Joint Surg [Br] 1997;79-B:403-11.

Received 27 August 1996; Accepted after revision 29 October 1996

H. Tsuchiya, MD, PhD, Associate Professor

K. Tomita, MD, PhD, Professor and Chairman

K. Minematsu, MD, Postdoctoral Fellow

Y. Mori, MD, Postdoctoral Fellow

N. Asada, MD, Postdoctoral Fellow

S. Kitano, MD, Postdoctoral Fellow

Department of Orthopaedic Surgery, School of Medicine, Kanazawa

University, 13-1 Takaramachi, Kanazawa 920, Japan.

Correspondence should be sent to Dr H. Tsuchiya.

(c)1997 British Editorial Society of Bone and Joint Surgery 0301-620X/97/37198\$2.00
There has been a dramatic improvement in the survival rate of patients with sarcomas and in the successful salvage of limbs as a result of progress in chemotherapy, radiological evaluation, surgical technique and the technology of materials and implants. Complications, however, such as deep infection, fracture, bone resorption, and breakage of prostheses still occur. ${ }^{1}$ The challenge to provide long-lasting survival and function of the limb after reconstruction is now being met with biological solutions using living bone. $^{2-7}$ The ideal reconstruction should have biological affinity, resistance to infection, sufficient biomechanical strength, and durability. Vascularised bone transfer has limitations in length and strength and since 1990 we have been using distraction osteogenesis (callotasis) which can regenerate bone of sufficient strength for reconstruction. We now report our findings.

\section{PATIENTS AND METHODS}

Our series included 19 patients with tumours of a limb: ten had an osteosarcoma (seven conventional, two central low-grade, and one parosteal), five had a giant-cell tumour, two a chondrosarcoma, one a Ewing's sarcoma, and one a malignant fibrous histiocytoma. Six patients with high-grade osteosarcomas treated with intra-arterial chemotherapy combined with caffeine ${ }^{8}$ had marginal excisions to preserve important structures such as the epiphysis, neurovascular bundles, and ligaments. Those with other malignant tumours underwent wide excision. Patients with giant-cell tumours had an en-bloc excision, thorough curettage at the subarticular area, and phenol cauterisation.

Distraction osteogenesis involved three different procedures: bone transport, shortening-distraction, or both combined with the use of an intramedullary nail. Two patients treated earlier by an autoclaved bone graft and one by a vascularised fibular graft had distraction osteogenesis after the failure of the primary operation.

Group 1. Ten patients, five with osteosarcoma and five with giant-cell tumour, with a mean age of 26.4 years had bone transport. A monofocal osteotomy was performed to allow a bone segment to be transported to fill the defect produced by tumour resection. Bone graft was taken from the iliac crest and placed at the docking site to facilitate union soon 
after the defect had been bridged. The tumours were in the proximal tibia in eight patients, the midshaft of the tibia in one and the distal femur in one. The average length of the defect was $8.8 \mathrm{~cm}$ (4.7 to 18 ). Three patients had chemotherapy during distraction osteogenesis.

Group 2. Two patients with osteosarcoma and one with Ewing's sarcoma were treated by shortening-distraction. We performed distraction osteogenesis at the remaining diaphysis after shortening of the metaphyseal defects. There was no need for an iliac bone graft at the original compression site when this was followed by distraction. The three tumours were in the proximal femur, the distal femur, and the proximal tibia. The mean age of the patients was 14.3 years and the mean length of the defects was $8.2 \mathrm{~cm}$ (5.5 to 13). Two patients had chemotherapy during distraction. Two children, one with an osteosarcoma and the other with Ewing's sarcoma, had the affected bone lengthened by an excess of $2 \mathrm{~cm}$ to correct anticipated discrepancy in leg length.

Group 3. This group was treated by bone transport or by shortening-distraction combined with an intramedullary nail to reduce the duration of external fixation (Fig. 1). Two techniques were used. When possible, distraction osteogenesis was performed after an intramedullary nail had been introduced with either proximal or distal locking screws. Screws were placed on the unlocked side after distraction was complete. If the remaining epiphysis was too thin to use an intramedullary nail or when part of the epiphysis was resected with the tumour, intramedullary nailing was performed after bone transport or shorteningdistraction had been completed. After removal of the external fixator and placement of the second set of locking screws, a cast or splint was applied until the regenerated callus had consolidated. This group included three patients with osteosarcoma, two with chondrosarcoma and one with malignant fibrous histiocytoma. Their mean age was 41.3 years and the mean length of the defects was $7.9 \mathrm{~cm}(3.5$ to 15). Two patients with osteosarcoma had chemotherapy during distraction. Two patients with pelvic chondrosarco-

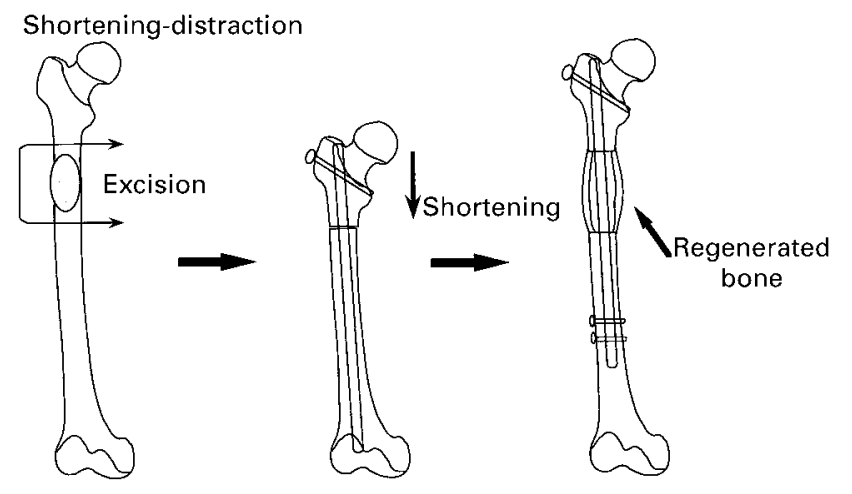

Fig. 1

Distraction osteogenesis over an intramedullary nail for shorteningdistraction. ma had resection of the hip with an arthrodesis and a standard tibial lengthening with an intramedullary nail was performed for inequality of leg length.

Distraction was begun approximately 7 to 14 days after the operation at $0.5 \mathrm{~mm}$ twice daily or $0.25 \mathrm{~mm}$ four times daily. This was later either reduced to zero when the callus formation was delayed or impaired or increased to $1.5 \mathrm{~mm}$ per day when the callus formation was likely to consolidate prematurely. The external fixator was removed when sufficient consolidation had been obtained and a cast or splint applied for approximately four weeks. When callus formation was poor, distraction was delayed or compression and distraction of a moving segment (the accordion manoeuvre) was applied.

Evaluation. Three indices were used to evaluate the results: an external fixation index obtained by dividing the entire duration of external fixation by the length of bone regeneration; a distraction index obtained by dividing the duration of distraction by the length of bone regeneration; and a maturation index calculated by dividing the duration of external fixation, measured from the completion of distraction to the removal of external fixation, by the length of bone regeneration. The function of the affected limb was assessed according to Enneking. ${ }^{9}$ The mean period of follow-up for the 19 patients was 37 months (13 to 68). All patients were followed up for more than two years except for two who died after 13 months.

\section{RESULTS}

In the bone-transport group, an Ilizarov external fixator was used in nine patients and a unilateral lengthener in one. The mean external fixation index was 39.5 days $/ \mathrm{cm}$ (27.9 to 63.5$)$, the distraction index 19.5 days/cm (8.5 to 33.8 ) and the maturation index 18.8 days/cm (1.5 to 44.4$)$. Seven complications occurred in six patients, including two skin invaginations, two pes equinus, one premature consolidation, one fracture at the docking site, and one subluxation of the head of the fibula. Skin invagination was treated by resection of elongated skin and suture. Pes equinus was managed by physiotherapy after removal of the Ilizarov apparatus. The apparatus was reapplied around the fracture at the docking site and the subluxation of the head of the fibula was reduced and fixed by screws during final removal of the Ilizarov fixator. The case of premature consolidation of regenerated bone was managed by a further percutaneous osteotomy, resulting in excellent bone regeneration. The function of the affected leg was rated excellent in eight patients, good in one and fair in one.

In the shortening-distraction group, an Ilizarov apparatus was used in all three patients. The mean external fixation index was 34.1 days/cm (32.7 to 34.8$)$, the distraction index 9.4 days/cm (7.3 to 10.8$)$ and the maturation index 23.4 days/cm (20.3 to 26.6). One patient with proximal tibial osteosarcoma had shortening of $5.5 \mathrm{~cm}$, and suffered skin 
LIMB SALVAGE USING DISTRACTION OSTEOGENESIS

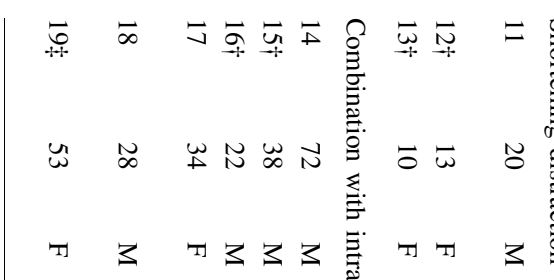

ठ

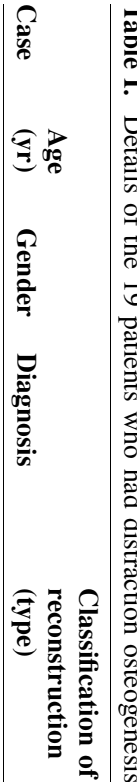

吾窟言:

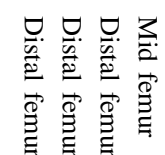

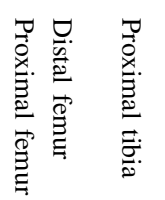

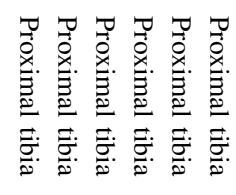

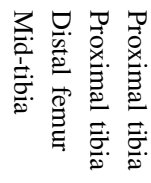

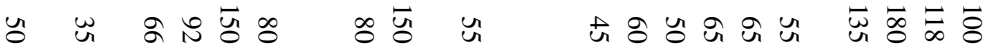

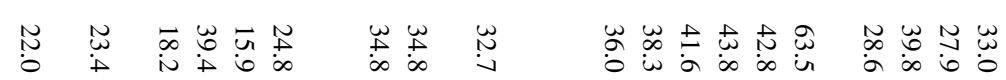

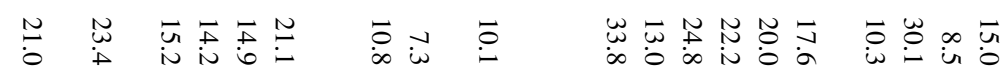

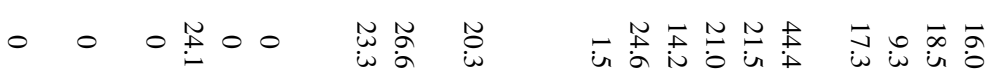

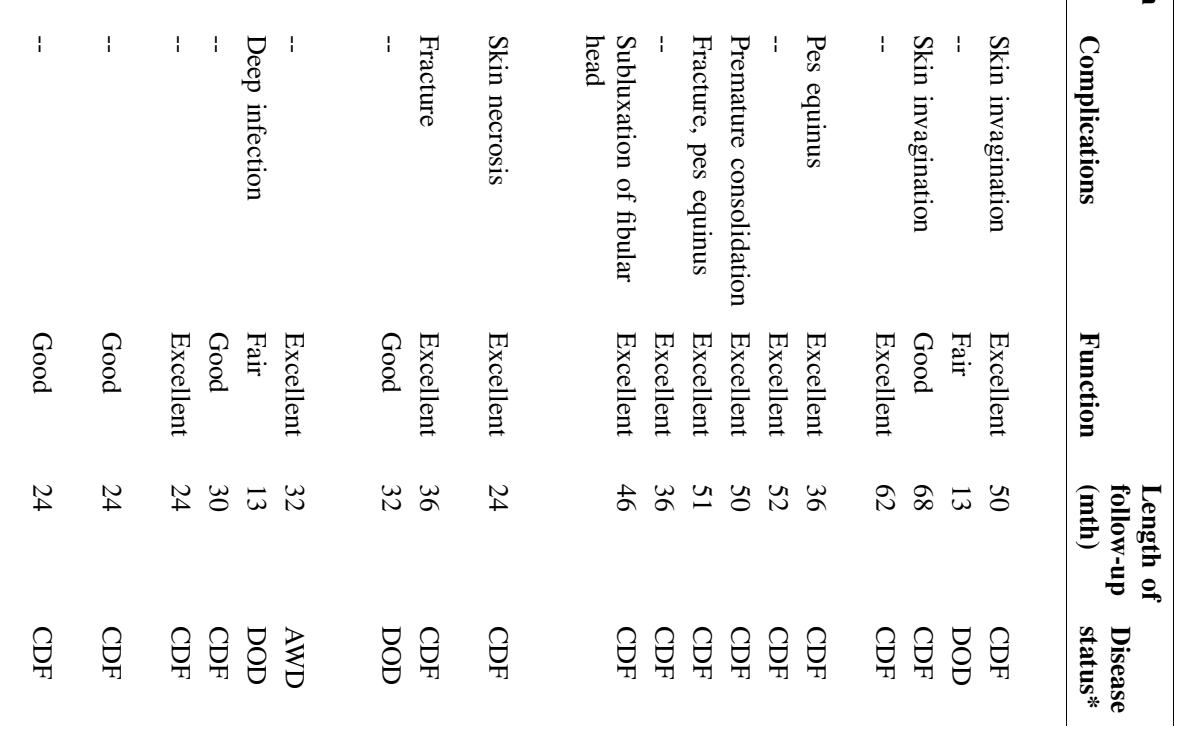



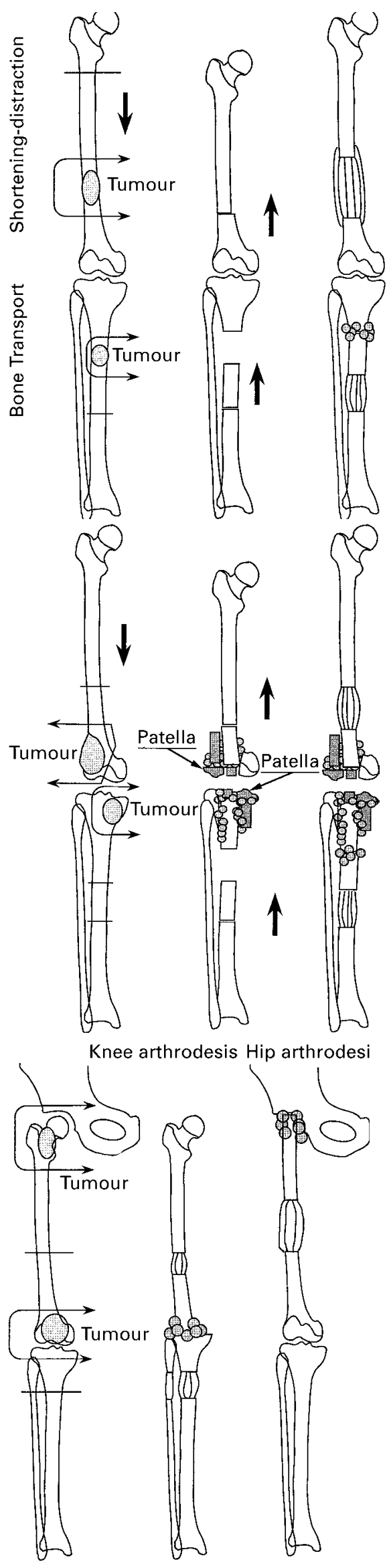

Fig. 2a
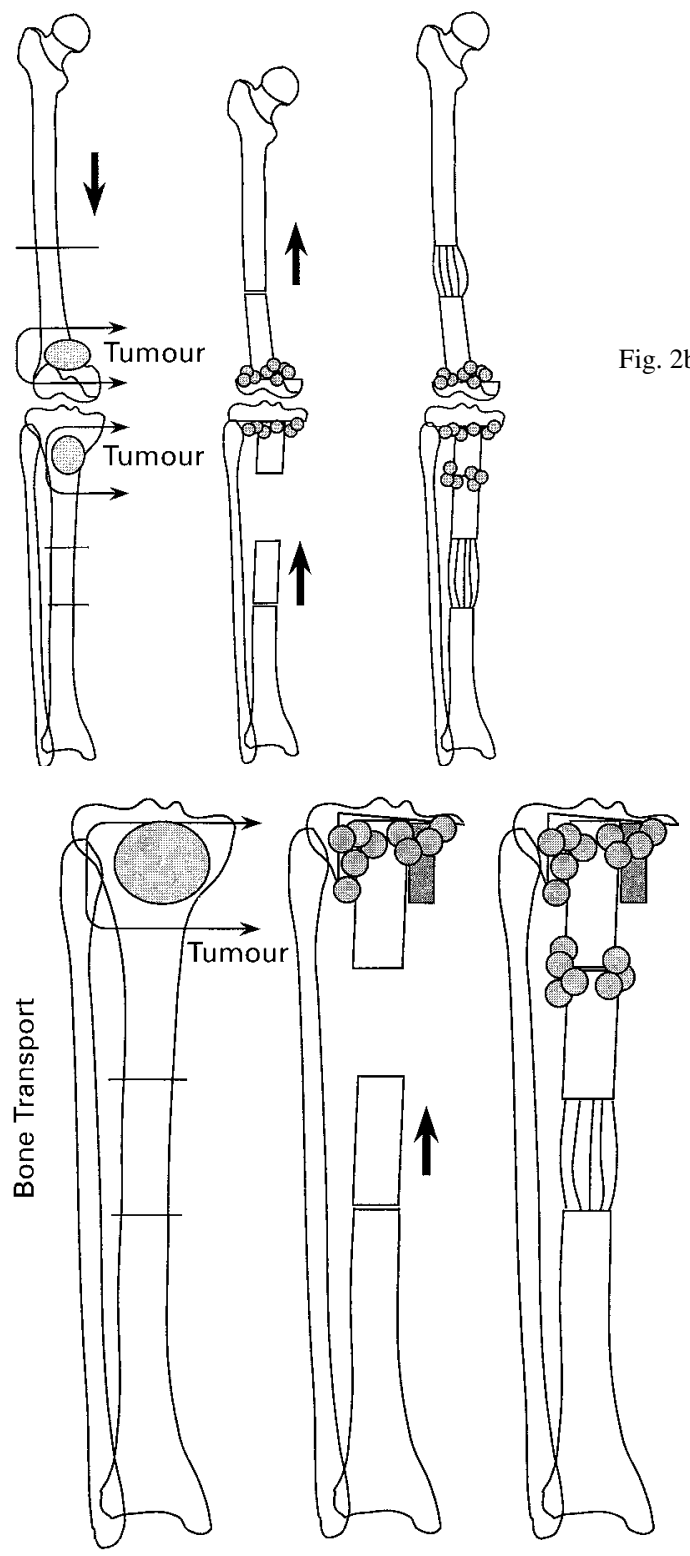

Fig. 2b

Fig. 2d

Fig. 2e

The classification of reconstruction by distraction osteogenesis using bone transport (shown in tibia) or shortening distraction (shown in femur): type 1 , diaphyseal reconstruction (a); type 2, metaphyseal reconstruction (b); type 3 , epiphyseal reconstruction (c); type 4, subarticular reconstruction (d); and type 5, arthrodesis (e). 


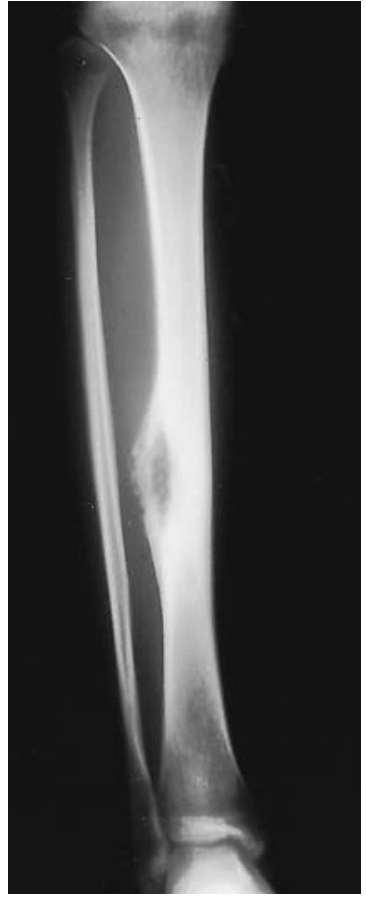

Fig. 3a

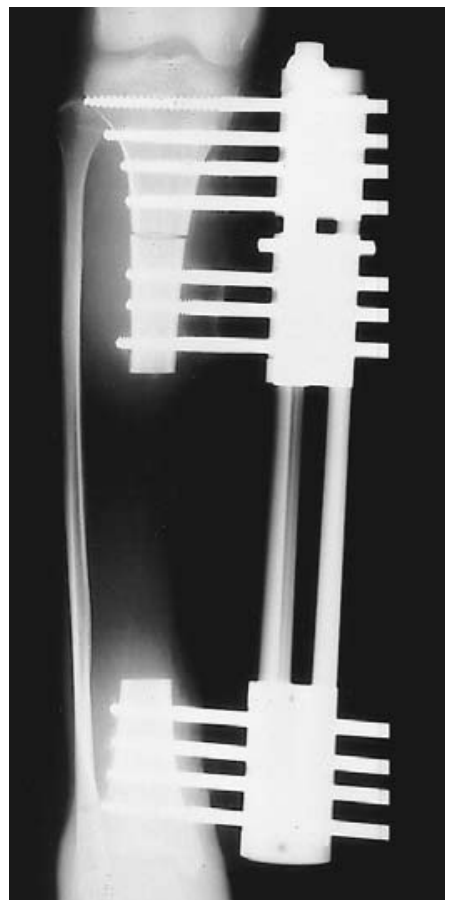

Fig. 3b

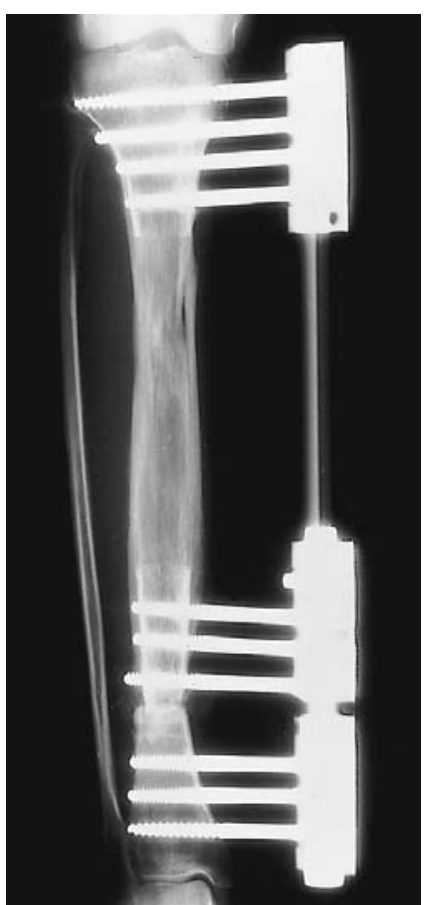

Fig. 3c

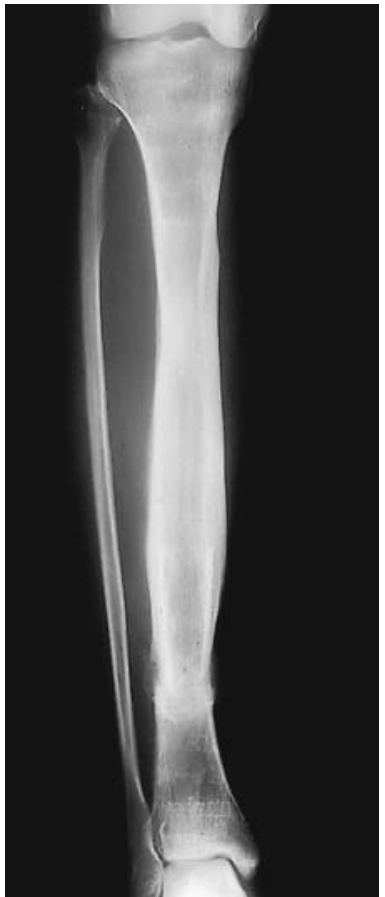

Fig. 3d

Case 4. Figure $3 \mathrm{a}$ - A low-grade osteosarcoma in the mid-tibia of a 15-year-old girl. Figure $3 \mathrm{~b}$ - After wide excision of the tumour, a unilateral lengthener was applied to fill a $13.5 \mathrm{~cm}$ defect using type-1 reconstruction. Figure $3 \mathrm{c}-$ At the end of transport. Figure $3 \mathrm{~d}-$ There is excellent bone formation and limb function 62 months after the initial operation.

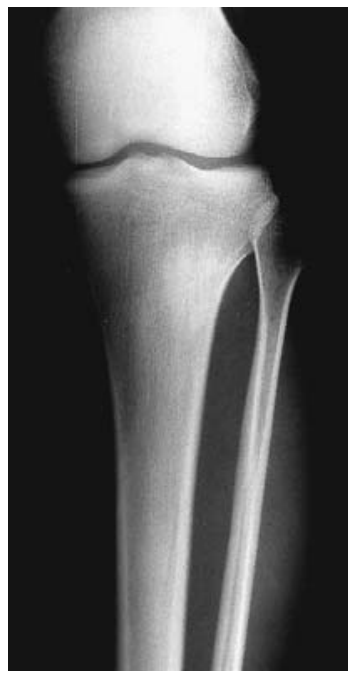

Fig. 4a

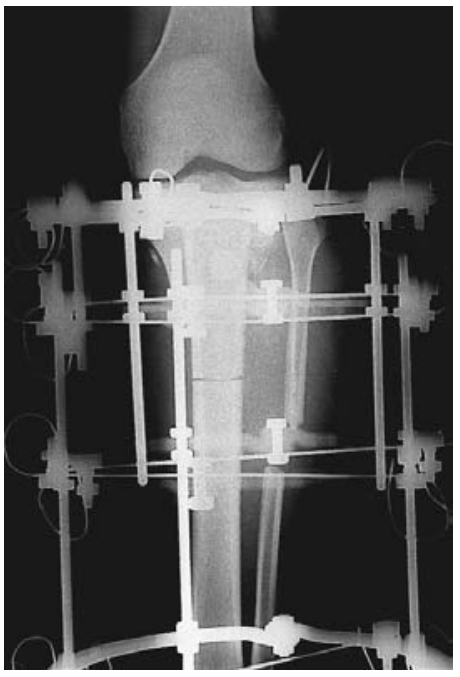

Fig. 4b

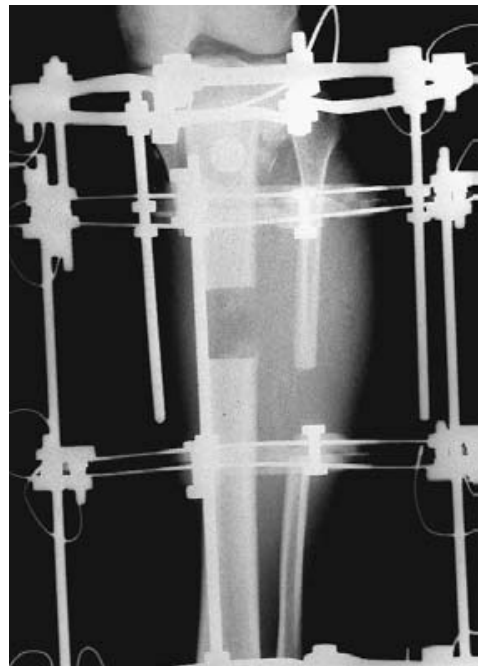

Fig. 4c

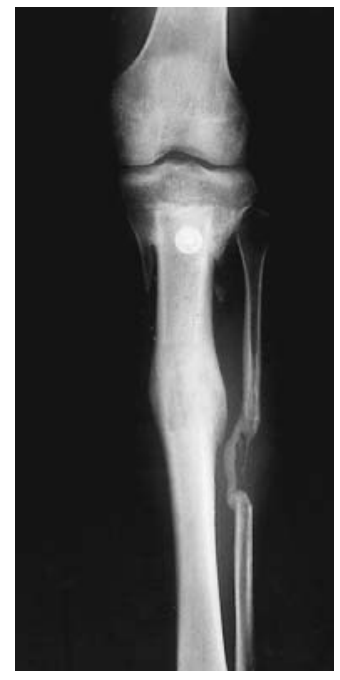

Fig. 4d

Case 11. Figure $4 \mathrm{a}$ - Radiograph of a low-grade osteosarcoma in the proximal tibia of a 20 -year-old man. Figure $4 \mathrm{~b}-$ After wide excision of the tumour, a type- 2 reconstruction was performed using an Ilizarov apparatus. The patellar ligament was reattached to the shortened diaphysis. Figure $4 \mathrm{c}-$ During distraction. Figure $4 d-$ Radiograph at 20 months after the initial operation.

necrosis after wound closure. This was treated conservatively, allowing the wound to granulate during the distraction phase. One patient with osteosarcoma in the distal femur sustained a fracture of the regenerated callus which was treated by a cast for six weeks. The function of the affected limb was rated excellent in two patients and good in the other.

In the group in which treatment was combined with an intramedullary nail, an Ilizarov apparatus was used for two patients and a unilateral lengthener for four. A RussellTaylor nail was used in five patients and a Huckstep nail in one. The mean external fixation index was 24.0 days $/ \mathrm{cm}$ 
(15.9 to 39.4) and the distraction index 18.3 days/cm (14.2 to 23.4). One patient developed a deep infection which was treated by removal of the lengthener, irrigation, and intravenous antibiotics when bone transport had been completed. The function of the affected limb was rated excellent in two patients, good in three, and fair in one (Table I).

The mean external fixation and the distraction indices for the seven patients who had chemotherapy during distraction were 35.6 days $/ \mathrm{cm}$ and 12.6 days/cm, respectively. In the 12 patients who did not receive chemotherapy these were 32.7 days $/ \mathrm{cm}$ and 20.4 days $/ \mathrm{cm}$. There was no statistically significant difference for the external fixation index ( $\mathrm{p}=0.56$, Student's $t$-test).

No local recurrence of tumour was seen. Fifteen patients are currently free from disease and one patient with a stageIIIB malignant fibrous histiocytoma is alive but has metas- tases. Two patients with stage-IIIB osteosarcoma and one with Ewing's sarcoma have died from metastatic disease. Classification of reconstruction with distraction osteogenesis (Fig. 2). We have classified reconstruction with distraction osteogenesis into five types based on the location of the defect after resection of the tumour.

Type 1. Diaphyseal reconstruction.

Type 2. Metaphyseal reconstruction.

Type 3. Epiphyseal reconstruction.

Type 4. Subarticular reconstruction.

Type 5. Arthrodesis.

In type 1 the diaphyseal defect is reconstructed by bone transport or shortening-distraction (Fig. 3). To reconstruct the metaphyseal defect in type 2 Ilizarov wires or half pins for fixation may be used through the remaining epiphysis. In addition to bone transport or shortening-distraction, the

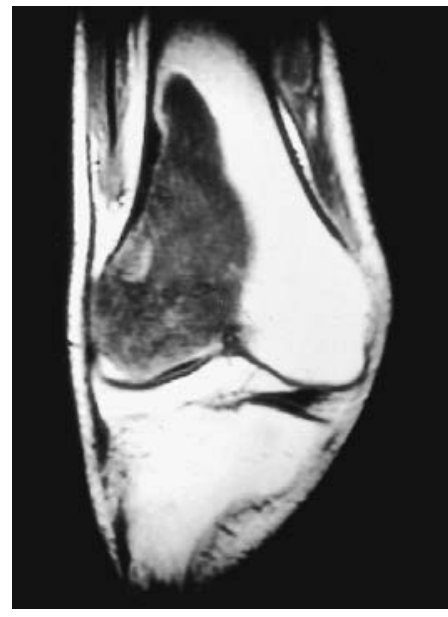

Fig. 5a

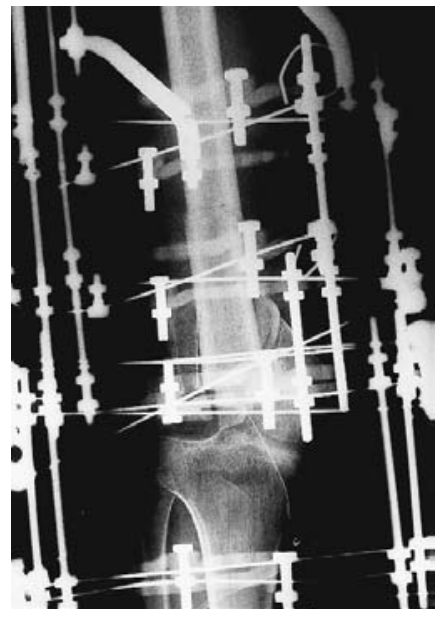

Fig. 5b

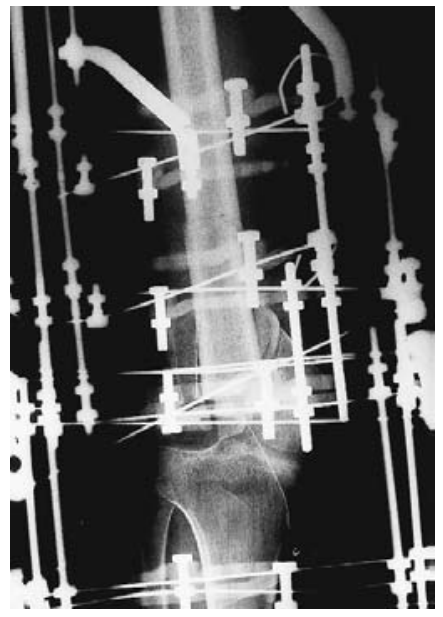

Fig. 5c

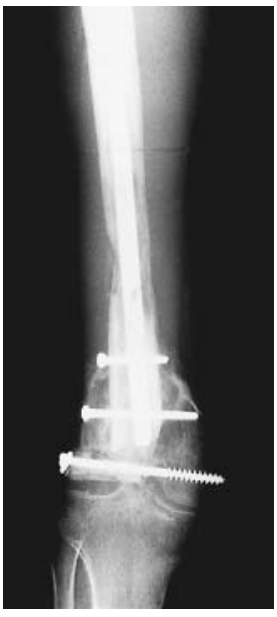

Fig. 5d

Case 16. Figure 5a - MRI of an osteosarcoma in the distal femur of a 22-year-old man. Figure 5b - After preoperative chemotherapy and marginal excision with preservation of the medial epiphysis and medial collateral ligament, a type- 3 reconstruction was performed and the lateral collateral ligament reconstructed using the iliotibial band. Figure $5 \mathrm{c}-$ Radiograph during distraction. Figure 5d - After the completion of distraction, an intramedullary nail was introduced. There is good function of the limb 30 months after the initial operation.

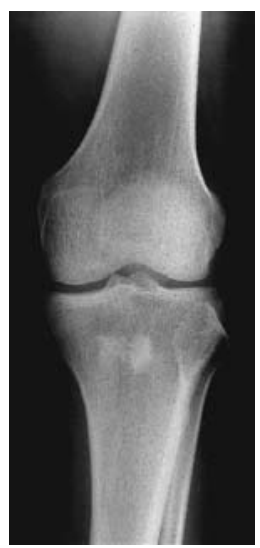

Fig. 6a

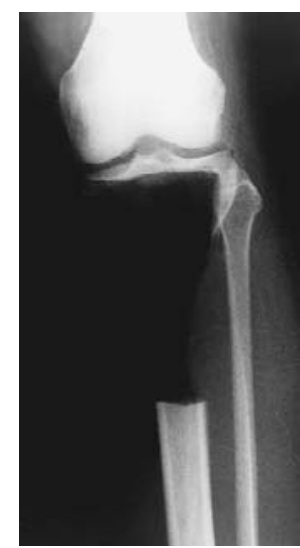

Fig. 6b

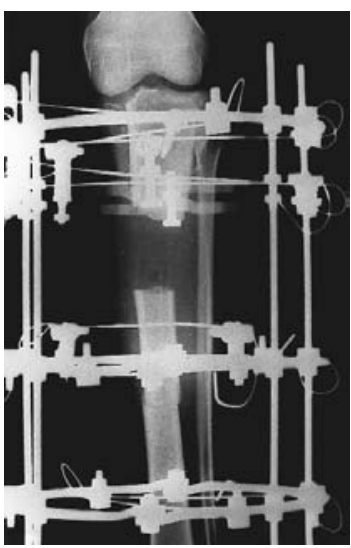

Fig. 6c

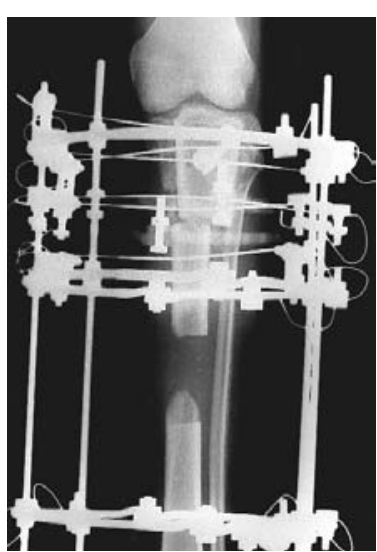

Fig. 6d

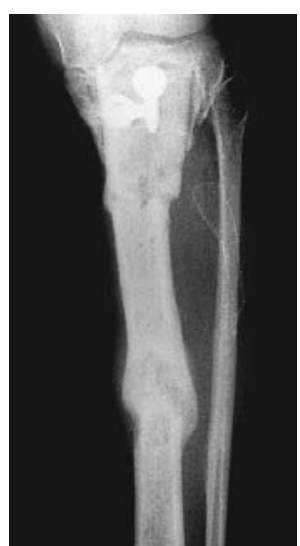

Fig. 6e

Case 5. Figure $6 \mathrm{a}$ - Radiograph of a conventional osteosarcoma in the proximal tibia of a 43 -year-old woman. Figures $6 \mathrm{~b}$ and $6 \mathrm{c}-$ Preoperative chemotherapy showed a good response and the articular cartilage and subchondral bone were preserved by a marginal procedure and a type-4 reconstruction. The patellar ligament was reattached to a bone cylinder from the diaphysis. Figure $6 \mathrm{~d}-$ Radiograph during distraction. Figure $6 \mathrm{e}-\mathrm{There}$ is excellent function of the knee 36 months after the initial operation. 


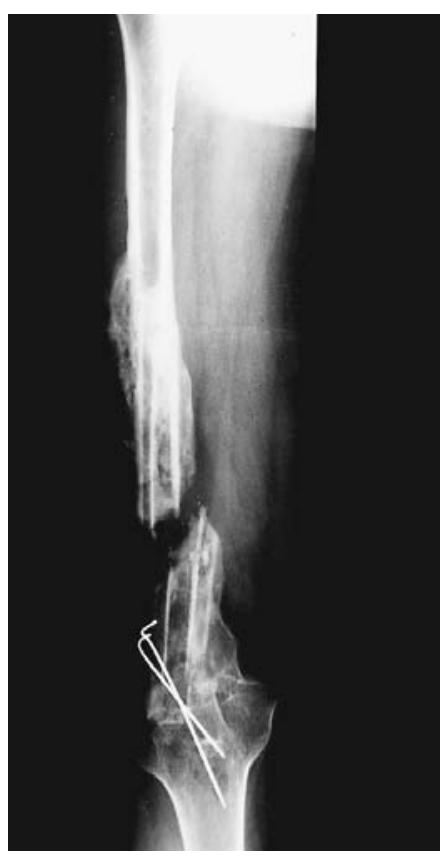

Fig. 7a

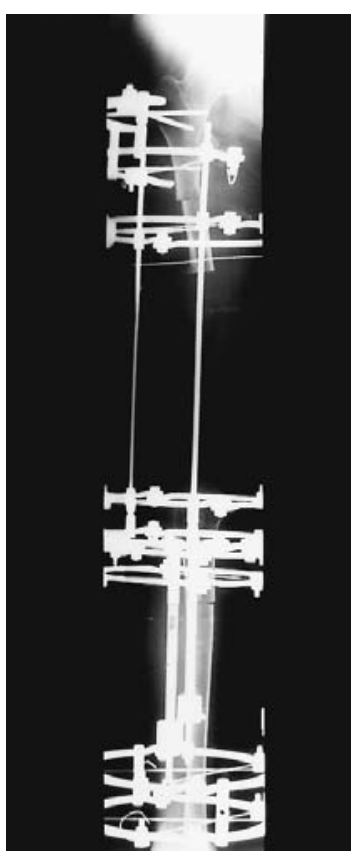

Fig. 7b

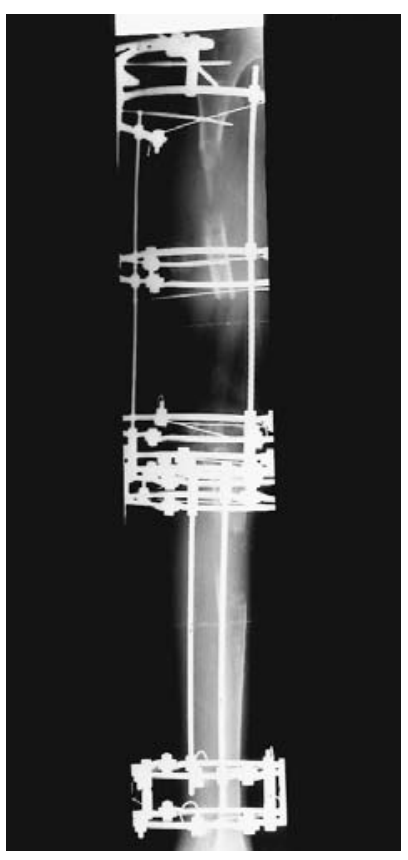

Fig. 7c

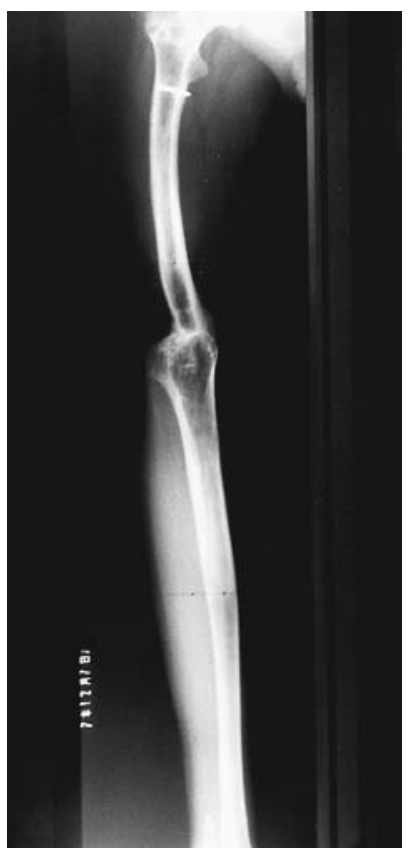

Fig. 7d

Case 3. Figure $7 \mathrm{a}$ - Radiograph of a conventional osteosarcoma in a 20 -year-old man. It was initially treated by a vascularised fibular graft at the age of 14 years, with failure due to repetitive fracture and infection. Figure $7 \mathrm{~b}-\mathrm{A}$ type- 5 reconstruction was performed with bone transport of $18 \mathrm{~cm}$ in the femur and tibial lengthening of $8 \mathrm{~cm}$. Figure $7 \mathrm{c}-$ Radiograph during distraction. Figure $7 \mathrm{~d}-$ Arthrodesis was complete two years after the initial operation.

metaphyseal defect may be reconstructed with a bone cylinder from the diaphysis, filling the defect by bone transport (Fig. 4). Type 3 includes defects of the metaphysis and unilateral defects of the epiphysis. Type-3 metaphyseal defects are reconstructed by shortening the diaphysis or by using a bone cylinder from the diaphysis. A unilateral defect of the epiphysis is repaired with the articular surface of the ipsilateral patella ${ }^{10}$ and a strut graft from the iliac crest. The diaphyseal defect is then filled in by bone transport. When the defect has been shortened, a standard lengthening procedure is performed at the diaphysis (Fig. 5).

The type- 4 procedure is used for reconstruction of subarticular defects. We have previously described this for the treatment of giant-cell tumours in the proximal tibia, ${ }^{11}$ and it may also be used at the distal tibia. Articular cartilage with or without some subchondral bone is left in situ after excision of the tumour, and Ilizarov wires cannot be applied through the epiphysis. A bone cylinder is taken from the diaphysis and fixed by wires to the remaining lateral or medial wall. The newly-created diaphyseal defect is then filled by bone transport (Fig. 6).

In type-5 procedures arthrodesis is used to reconstruct defects after joint resection and to correct subsequent discrepancy of leg length (Fig. 7).

A unilateral external fixator is convenient for a type-1 reconstruction and for diaphyseal lengthening in a type-5 arthrodesis. An Ilizarov apparatus is useful for the fixation of epiphyseal or metaphyseal structures and for simultaneous bone lengthening in the management of reconstructions of types 2,3 and 4.

We used the type- 1 procedure in two patients, type 2 in six, type 3 in two, type 4 in six and type 5 in three. The mean external fixation index was 26.7 days/cm for type 1 , 28.2 for type 2, 33.7 for type 3, 44.2 for type 4 , and 28.4 for type 5 .

\section{DISCUSSION}

Distraction osteogenesis has been widely used for the treatment of leg-length discrepancy, nonunion, traumatic bone defects, deformity, and osteomyelitis. ${ }^{12-14}$ Its use in the management of musculoskeletal tumours has not been studied in detail. It has been employed in the reconstruction of diaphyseal defects after excision of tumours and for arthrodesis after joint resection. ${ }^{3,4,6}$ Cañadell et al $^{15}$ used distraction osteogenesis to expand the tumour-free margin and to preserve the epiphysis by physeal distraction.

We have used distraction osteogenesis to reconstruct defects in the metaphysis and epiphysis as well as in the diaphysis. We classified the procedures available into five types. Types 2, 3 and 4 were most often used, because tumours occur more often in a periarticular location. For high-grade sarcomas, type-2, type- 3 and type- 4 reconstructions are indicated for patients in whom excision of the tumour can be performed under effective preoperative chemotherapy in order to reduce the risk of local recurrence. Caffeine-assisted chemotherapy, ${ }^{8}$ which has resulted in a high rate of local control, has allowed minimalisation of the 
resection of healthy tissue and periarticular reconstruction for osteosarcoma. Joint preservation and periarticular reconstruction should be limited to those who respond well to preoperative chemotherapy and are expected to have total tumour necrosis on radiological evaluation or biopsy. The technique should not be used in those who respond poorly, because local recurrence will lead to a poor prognosis.

The external fixation index for type-4 subarticular reconstruction was significantly greater than for the other types because free, devitalised diaphyseal bone had been used to reconstruct the subarticular defect and it was difficult to apply intramedullary nailing. The type-3 reconstruction using an ipsilateral patella to replace the articular surface may result in osteoarthritic changes later, although a low incidence of this has been reported in the management of giant-cell tumours. ${ }^{10}$

When maturation of regenerated bone is very slow, decreasing the distraction speed, delaying distraction, axial shortening, ${ }^{16}$ or direct-current stimulation ${ }^{17}$ may be used. The addition of an intramedullary nail ${ }^{18}$ is beneficial as a temporary measure. The combination of an intramedullary nail and distraction osteogenesis may reduce the period of treatment with a decrease in the incidence of wire or pintrack infections. The external fixation index can be reduced as the maturation index approaches zero. We therefore apply intramedullary nailing for distraction osteogenesis whenever feasible. A thin, stress-resistant intramedullary nail is appropriate for combined use to preserve the intramedullary blood supply and allow space for the insertion of wires or half pins. If shortening-distraction is applied, bone graft at the docking site is not necessary. Femoral shortening of about 10 to $15 \mathrm{~cm}$ can be achieved if wound closure is feasible. Careful attention should be paid to the shortening of the tibia because of circulatory disturbance, but tibial defects can be satisfactorily treated by bone transport for extensive bone loss.

We performed distraction osteogenesis on seven patients who were having chemotherapy after the operation. Although this was expected to delay bone regeneration initially and to increase the distraction index, callus was successfully distracted at about $0.8 \mathrm{~mm}$ per day in our series. Only one patient had a deep infection. In patients with osteosarcoma, minimalisation of the surgical margin aimed at the preservation of healthy tissue with effective chemotherapy may give satisfactory results. The number of patients was small, however, and the effects of chemotherapy on distraction osteogenesis or on bone regeneration are still unknown.

Bony defects created by limb-salvage procedures may be reconstructed by a variety of methods but the incidence of complications such as fracture, nonunion, deformity, and infection is high, ${ }^{19-25}$ and is directly related to the size of the graft and the use of chemotherapy. ${ }^{20}$ The use of allografts or autoclaved bone in combination with vascularised bone grafts has been subjected to preliminary trials. ${ }^{26,27}$ Even although we encountered ten complications in 19 patients all were managed successfully.

The living bone regenerated in distraction osteogenesis will eventually provide sufficient biomechanical strength, stability and durability. Although external fixation may be prolonged, reconstruction by distraction osteogenesis results in a stable leg, avoids complications associated with prosthetic or allograft replacement and provides attachment for ligaments, tendons, and muscles. In growing children, leg-length discrepancies may be corrected by distraction osteogenesis after they have matured or during the distraction after tumour excision. Expandable prostheses have been developed for this problem. ${ }^{28}$

Distraction osteogenesis is beneficial for patients with a good long-term prognosis and for growing children. For patients with metastatic disease, prosthetic replacement after resection of the tumour will provide an early improvement in function and is indicated. Other techniques offer simpler and less time-consuming reconstruction for some bony defects, but distraction osteogenesis has a place in reconstruction after the excision of a tumour; it will become more attractive if the duration of the treatment can be shortened.

No benefits in any form have been received or will be received from a commercial party related directly or indirectly to the subject of this article.

\section{REFERENCES}

1. Tsuchiya H, Tomita K. Prognosis of osteosarcoma treated by limb salvage surgery: the ten-year intergroup study in Japan. Jpn J Clin Oncol 1992;22:347-53.

2. Han C-S, Wood MB, Bishop AT, Cooney WP III. Vascularised bone transfer. J Bone Joint Surg [Am] 1992;74-A:1441-9.

3. Nakatsuka Y, Ozaki T, Kawai A, Akazawa H, Inoue H. Reconstruction of bony defect after resection of a high grade osteosarcoma using bone transport technique: a case report. Proceedings of 7th International Symposium on Limb Salvage, Singapore, 1993;596.

4. Stoffelen D, Lammens J, Fabry G. Resection of a periosteal osteosarcoma and reconstruction using the Ilizarov technique of segmental transport. J Hand Surg [Br] 1993;18:144-6.

5. Tsuchiya H, Tomita K, Tsubota S, Shinokawa Y, Katsuo S, Tokuumi Y. Treatment of bone and soft tissue tumours using external fixators. Proceedings of 7th International Symposium on Limb Salvage, Singapore, 1993:137-43.

6. Said GZ, El-Sherif EK. Resection-shortening-distraction for malignant bone tumours: a report of two cases. J Bone Joint Surg [Br] 1995;77-B:185-8.

7. Usui M, Ishii S, Naito T, Wada T, Yamawaki S, Isu K. Limb-saving surgery in osteosarcoma by vascularised fibular graft. J Orthop Sci 1996;1:4-10.

8. Tsuchiya H, Tomita $\mathbf{K}$, Sugihara $\mathbf{M}$, et al. Intra-arterial chemotherapy combined with caffeine and conservative surgery for osteosarcoma. In: Rao RS, Deo MG, Sanghvi LD, eds. Proceedings of the XVI International Cancer Congress, Monduzzi Editore, Bologna, 1994:2453-6.

9. Enneking WF. A system for functional evaluation of the surgical management of musculoskeletal tumors. In: Enneking WF, ed. Limb salvage in musculoskeletal oncology. New York: Churchill Livingstone, 1987:5-16.

10. Campanacci M, Cervellati C, Donati U. Autogenous patella as replacement for a resected femoral or tibial condyle: a report on 19 cases. J Bone Joint Surg [Br] 1985;67-B:557-63.

11. Tsuchiya H, Tomita K, Shinokawa $Y$, et al. The Ilizarov method in the management of giant-cell tumours of the proximal tibia. $J$ Bone Joint Surg [Br] 1996;78-B:264-9.

12. Ilizarov GA, Deviatov AA. Operative elongation of the leg with simultaneous correction of the deformities. Ortop Travmatol Protez 1969;30:32-7. 
13. De Bastiani G, Aldegheri R, Renzi-Brivio L, Trivella G. Limb lengthening by callus distraction (callotasis). J Pediatr Orthop 1987; 7:129-34.

14. Ilizarov GA. Clinical application of the tension-stress effect for limb lengthening. Clin Orthop 1990;250:8-26.

15. Cañadell J, Forriol F, Cara JA. Removal of metaphyseal bone tumours with preservation of the epiphysis: physeal distraction before excision. J Bone Joint Surg [Br] 1994;76-B:127-32.

16. Hamanishi C, Yoshii T, Totani Y, Tanaka S. Lengthened callus activated by axial shortening: histological and cytomorphometrical analysis. Clin Orthop 1994;307:250-4.

17. Hamanishi C, Kawabata T, Yoshii T, Tanaka S. Bone mineral density changes in distracted callus stimulated by pulsed direct electrical current. Clin Orthop 1995;312:247-52.

18. Raschke MJ, Mann JW, Oedekoven G, Claudi BF. Segmental transport after unreamed intramedullary nailing: preliminary report of a 'monorail' system. Clin Orthop 1992;282:233-40.

19. Harrington KD, Johnston JO, Kaufer HN, Luck JV Jr, Moore TM. Limb salvage and prosthetic joint reconstruction for low-grade and selected high-grade sarcomas of bone after wide resection and replacement by autoclaved autogeneic grafts. Clin Orthop 1986; 211:180-214.

20. Gebhardt MC, Mankin HJ. The use of proximal tibial allografts in the reconstruction of tumors and other defects. In: Yamamuro T, ed. New developments for limb salvage in musculoskeletal tumors. Tokyo, Springer-Verlag, 1989:573-83.
21. Harris IE, Leff AR, Gitelis S, Simon MA. Function after amputation, arthrodesis, or arthroplasty for tumors about the knee. J Bone Joint Surg [Am] 1990;72-A:1477-85.

22. Gitelis S, Piasecki P. Allograft prosthetic composite arthroplasty for osteosarcoma and other aggressive bone tumours. Clin Orthop 1991; 270:197-201.

23. Brien EW, Terek RM, Healey JH, Lane JM. Allograft reconstruction after proximal tibial resection for bone tumours: an analysis of function and outcome comparing allograft and prosthetic reconstructions. Clin Orthop 1994;303:116-27.

24. Clohisy DR, Mankin HJ. Osteoarticular allografts for reconstruction after resection of a musculoskeletal tumor in the proximal end of the tibia. J Bone Joint Surg [Am] 1994;76-A:549-54.

25. Tsuchiya H, Tomita K. Joint reconstruction utilizing autoclaved bone grafting. J Joint Surg 1996;15:53-60.

26. Manfrini M, Capanna R, Ceruso M, et al. Vascularised fibular autogrft as medial support of massive allografts after femur resections for bone tumours (preliminary results). Proceedings of 7 th International Symposium on Limb Salvage, Singapore, 1993:155-9.

27. Taguchi Y, Pho WHO, Kour AK, et al. Limb salvage method using autoclaved autograft supplemented with vascularised fibular graft. Proceedings of 7th International Symposium on Limb Salvage, Singapore, 1993:161-5.

28. Lewis MM. The use of an expandable and adjustable prosthesis in the treatment of childhood malignant bone tumors of the extremity. Cancer 1986;57:499-502. 